\title{
Aggregation of implication functions
}

\author{
Tomasa Calvo $^{1}$ Javier Martín $^{2}$ Gaspar Mayor ${ }^{2}$ \\ ${ }^{1}$ University of Alcalá de Henares \\ ${ }^{2}$ University of the Balearic Islands
}

\begin{abstract}
This paper deals with the problem of the aggregation of implication functions. After characterizing those binary operations which merge two implication functions into a new implication function, we study some aspects of the aggregated implication functions in terms of the two given ones. In particular, we are interested in aggregation processes such that properties of the implication functions are preserved.
\end{abstract}

Keywords: Fuzzy logic, Fuzzy implication, Implication function, Aggregation function.

\section{Introduction}

A many-valued propositional logic in which the set of truth values is modeled by the real unit interval $[0,1]$, and which is an extension of the classical Boolean logic, i.e., the logic with truth values $\{0,1\}$, is often called a fuzzy logic. In such a logic, the (fuzzy) implication If $p$, then $q$ with $p$ and $q$ fuzzy propositions, is usually interpreted by an implication function, that is, a function $I:[0,1] \times[0,1] \rightarrow$ $[0,1]$ which satisfies a set of natural axioms. A complete study of this class of functions can be found in [1] and references therein. Specifically, given a fuzzy set $A$ on an universe $X$, a fuzzy set $B$ on an universe $Y$, and points $x \in X, y \in Y$ one consider the fuzzy implication

$$
(A \rightarrow B)(x, y)=I(A(x), B(y))
$$

where $I$ is an implication function. Note that the value $(A \rightarrow B)(x, y)$ only depends, once fixed $I$, on the values taken by $A$ and $B$ at the points $x$ and $y$; we say that the fuzzy implication $\rightarrow$ is functionally expressible through the implication function I. Alternatively, one can consider fuzzy implications, as well as conjunctions, disjunctions and other logical connectives, defined in an axiomatic way (see [4]) by considering similar conditions to those used in the definition of implication function, but now applied directly on the fuzzy sets.

Approximate reasoning refers to processes by which imprecise conclusions are inferred from imprecise premises. When imprecision is fuzzy in nature, the term fuzzy reasoning is used.

The most common formal deduction process in fuzzy reasoning has the following structural pattern: if we know that $A$ implies $B$ and we know $A$, then we conclude $B$,

where $A$ and $B$ are fuzzy sets on universes $X$ and $Y$ respectively. This pattern of reasoning is called Modus Ponens $(M P)$. A modified version of the $M P$ is:

if we know that $A$ implies $B$ and we know $A^{\prime}$, then we conclude $B^{\prime}$,

$$
\text { (in short, } A \rightarrow B \& A^{\prime} / B^{\prime} \text { ) }
$$

where $A, A^{\prime}$ are fuzzy sets on $X$ and $B, B^{\prime}$ are fuzzy sets on $Y$, and the conclusion $B^{\prime}$ is given by the formula $B^{\prime}(y)=\sup \left\{T\left(A^{\prime}(x), I(A(x), B(y))\right) ; x \in\right.$ $X\}, y \in Y$, where the conjunction \& is modelled by a t-norm $T$ and the implication $\rightarrow$ is interpreted by an implication function $I$. The t-norm $T$ and the implication function $I$ are assumed such that $B^{\prime}=B$ when $A^{\prime}=A$; in this sense, this extended pattern of reasoning is called the Generalized Modus Ponens $(G M P)$.

In this paper, we address the aggregation of implication functions. More specifically, our purpose is to find functions $F:[0,1]^{n} \rightarrow[0,1]$ such that for any implication functions $I_{1}, \ldots, I_{n}, K=F\left(I_{1}, \ldots, I_{n}\right)$ it is also an implication function, where $K(x, y)=$ $F\left(I_{1}(x, y), \ldots, I_{n}(x, y)\right), x, y \in[0,1]$.

The paper is organized as follows. In the next section we give some basic preliminaries that will be used along the paper. In Section 3 we deal with the problem of aggregation of implication functions, and in the Section 4 we study the preservation of properties concerning implication functions through the process of aggregation. The paper ends with a section where some conclusions are mentioned.

\section{Preliminaries}

Here, we recall some basic definitions:

Definition 2.1 A map $F:[0,1]^{2} \rightarrow[0,1]$ is said to be an aggregation function if it satisfies:

i) (Monotonicity) $F(x, y) \leqslant F\left(x^{\prime}, y^{\prime}\right)$, whenever $x \leqslant x^{\prime}$ and $y \leq y^{\prime}$.

ii) (Boundary conditions) $F(0,0)=0$ and $F(1,1)=1$.

Definition 2.2 A triangular norm (t-norm for short) is an increasing mapping $T:[0,1]^{2} \rightarrow[0,1]$ which is associative, commutative, and has neutral 
element 1. A t-conorm is an increasing mapping $S:[0,1]^{2} \rightarrow[0,1]$ which is associative, commutative, and has neutral element 0 .

Definition 2.3 A negation is a decreasing mapping $N:[0,1] \rightarrow[0,1]$ such that $N(1)=0$ and $N(0)=1$. We say that a negation is strong if it is involutive.

The classical negation is $N(x)=1-x$.

A good reference book on t-norms is [2]. For aggregation functions see, for example, [3].

\section{Aggregation of implication functions}

Definition 3.1 A mapping $I:[0,1]^{2} \rightarrow[0,1]$ is said to be an implication function if $I(0,0)=$ $I(1,1)=1, I(1,0)=0$ and is decreasing w.r.t the first variable and increasing w.r.t. the second variable.

We will denote the set of implication functions defined on $[0,1]$ by $\mathcal{I F}$.

Proposition 3.2 Let $I, J \in \mathcal{I F}$ and a function $F:[0,1]^{2} \rightarrow[0,1]$. Then,

$$
K(a, b)=F(I(a, b), J(a, b))
$$

is an implication function, for all $I, J$, if and only if $F$ is an aggregation function.

Proof. First, we will see that if $F$ is an aggregation function, then $K$ is an implication function, for all $I, J$.

i) (Boundary conditions)

$$
\begin{aligned}
& K(0,0)=F(I(0,0), J(0,0))=F(1,1)=1 \\
& K(1,1)=F(I(1,1), J(1,1))=F(1,1)=1, \\
& K(1,0)=F(I(1,0), J(1,0))=F(0,0)=0 .
\end{aligned}
$$

ii) (Monotonicity) $K$ is decreasing with respect to the first variable: if $x \leqslant x^{\prime}$, then $I(x, y) \geqslant$ $I\left(x^{\prime}, y\right)$ and $J(x, y) \geqslant J\left(x^{\prime}, y\right)$.

Furthermore, $\quad F(I(x, y), J(x, y)) \geqslant$ $F\left(I\left(x^{\prime}, y\right), J\left(x^{\prime}, y\right)\right)$, and so $K(x, y) \geqslant K\left(x^{\prime}, y\right)$. Similarly, for the increasingness of $K$ with respect to the second variable.

Therefore $K$ is an implication function.

Now, we assume that $K \in \mathcal{I F}$, for all $I, J$, and we will show that $F$ is an aggregation function:

i) (Boundary conditions) From the boundary conditions of $K$ and for any fixed $I, J \in \mathcal{I F}$ we have

(a)

$$
\begin{aligned}
1 & =K(0,0) \\
& =F(I(0,0), J(0,0)) \\
& =F(1,1) .
\end{aligned}
$$

$$
\begin{aligned}
0 & =K(1,0) \\
& =F(I(1,0), J(1,0)) \\
& =F(0,0) .
\end{aligned}
$$

ii) (Monotonicity) If $a \leqslant a^{\prime}$ we need to prove that $F(a, b) \leqslant F\left(a^{\prime}, b\right)$. It is sufficient to find $x, x^{\prime}, y \in[0,1]$, with $x \geqslant x^{\prime}$, and $I, J \in \mathcal{I F}$, such that

$$
\begin{aligned}
& I(x, y)=a, \\
& I\left(x^{\prime}, y\right)=a^{\prime}, \\
& J(x, y)=J\left(x^{\prime}, y\right)=b .
\end{aligned}
$$

For that, we consider these implication functions:

$$
I(x, y)= \begin{cases}1 & \text { if } x=0 \text { or } y=1 \\ a^{\prime} & \text { if } 0<x \leqslant y<1 \\ 0 & \text { if }(x, y)=(1,0) \\ a & \text { otherwise }\end{cases}
$$

and

$$
J(x, y)= \begin{cases}1 & \text { if } x=0 \text { or } y=1 \\ 0 & \text { if }(x, y)=(1,0) \\ b & \text { otherwise. }\end{cases}
$$

Then,

$$
\begin{aligned}
F(a, b) & =F(I(x, y), J(x, y))) \\
& =K(x, y) \\
& \leqslant K\left(x^{\prime}, y\right) \\
& =F\left(I\left(x^{\prime}, y\right), J\left(x^{\prime}, y\right)\right) \\
& =F\left(a^{\prime}, b\right)
\end{aligned}
$$

that is, $F(a, b) \leqslant F\left(a^{\prime}, b\right)$.

Analogously, if $b \leqslant b^{\prime}$ we need to prove $F(a, b) \leqslant F\left(a, b^{\prime}\right)$. For this, we take now the following implication functions:

$$
I(x, y)= \begin{cases}1 & \text { if } x=0 \text { or } y=1 \\ 0 & \text { if }(x, y)=(1,0) \\ a & \text { otherwise }\end{cases}
$$

and

$$
J(x, y)=\left\{\begin{array}{ll}
1 & \text { if } x=0 \text { or } y=1 \\
b^{\prime} & \text { if } 0<x \leqslant y<1 \\
0 & \text { if }(x, y)=(1,0) \\
b & \text { otherwise, }
\end{array},\right.
$$

and then we proceed in a similar way.

If $\mathrm{F}$ is commutative then the aggregated of two implication functions does not depend on the order in which they are taken. On the other hand, in order to aggregate more than two implication functions the associativity of the aggregation function $F$ is needed.

Example 3.3 Given $f:[0,1] \rightarrow[0,1]$ we consider $F:[0,1]^{2} \rightarrow[0,1]$ defined by $F(a, b)=f(a)$. Then $F$ is an aggregation function if and only if $f$ is increasing, with $f(0)=0$ and $f(1)=1$. Of course, in this case $K(x, y)=F(I(x, y), J(x, y))=f(I(x, y))$ $i s$ the $f$-transformed of the first implication $I$.

Moreover, $F$ is associative if and only $f^{2}=f$.

Note that the only increasing continuous function satisfying $f^{2}=f$ is $f=I d$. The shape of non continuous functions such that $f^{2}=f$ is as the function in Fig. 1. 


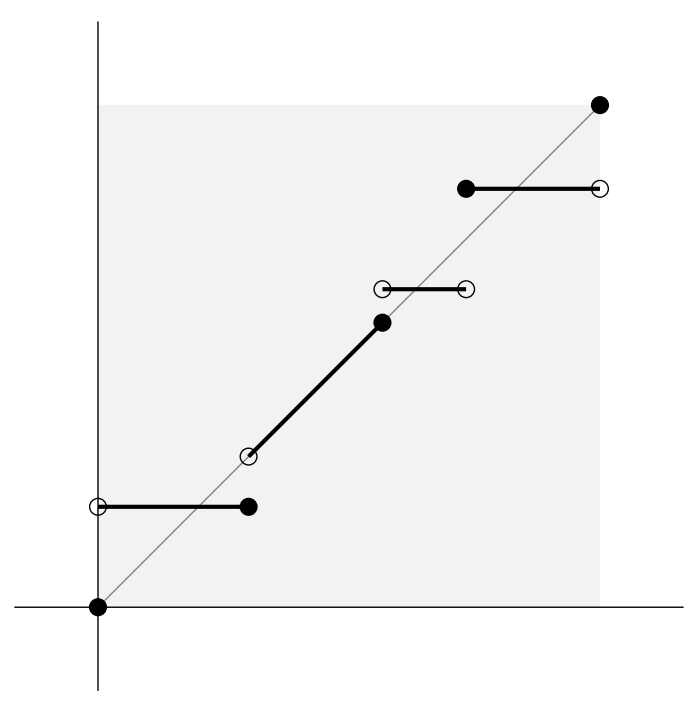

Figure 1: A non-continuous function such that $f^{2}=f$.

It could be interesting to study how properties of $F$ determine some features of $F(I, J)$. Let us denote by $L_{c}, c \in[0,1]$, the $c$-region of a function $L$, that is,

$$
L_{c}=\left\{(x, y) \in[0,1]^{2} ; L(x, y)=c\right\} .
$$

We will focus our attention on the meaning of idempotent, absorbent and neutral elements of $F$ in the result of the aggregation.

For example, the existence of idempotent elements, that is, elements $a$ such that $F(a, a)=a$.

Proposition 3.4 Let $F$ be an aggregation function. Then:

i) $F(I, J)_{a} \supseteq I_{a} \cap J_{a}$ for any $I, J \in \mathcal{I F}$ if and only if $a$ is an idempotent element of $F$.

ii) The equality $F(I, J)_{a}=I_{a} \cap J_{a}$ holds for any $I, J \in \mathcal{I F}$ if and only if it is $F(x, y)=a$ only at $(a, a)$.

\section{Proof.}

i) Let $a$ be an idempotent element of $F$ and suppose $(x, y) \in I_{a} \cap J_{a}$, that is, $I(x, y)=J(x, y)=$ a. Then,

$$
\begin{aligned}
F(I, J)(x, y) & =F(I(x, y), J(x, y)) \\
& =F(a, a) \\
& =a .
\end{aligned}
$$

and so, $(x, y) \in F(I, J)_{a}$.

The converse is as follows: we choose $I$ and $J$ such that $I_{a} \cap J_{a} \neq \emptyset$ and let $(x, y) \in I_{a} \cap J_{a}$. As $(x, y) \in F(I, J)_{a}$, we have

$$
\begin{aligned}
a & =F(I, J)(x, y) \\
& =F(I(x, y), J(x, y)) \\
& =F(a, a) .
\end{aligned}
$$

ii) From right-to-left we need to prove the inclusion $F(I, J)_{a} \subseteq I_{a} \cap J_{a}$.
If $(x, y) \in F(I, J)_{a}$, then $F(I(x, y), J(x, y))=$ $a$, but this holds only for $I(x, y)=J(x, y))=a$, that is, $(x, y) \in I_{a} \cap J_{a}$.

Now, the converse. Consider $F(x, y)=a=$ $F(I(z, t), J(z, t))$, where $(z, t) \in[0,1]^{2}$ and $I, J$ are implication functions such that $I(z, t)=x$ and $J(z, t)=y$. As $(z, t) \in I_{a} \cap J_{a}$, that is, $I(z, t)=J(z, t)=a$, we have $x=y=a$.

Remember that 0 and 1 are idempotent; so, these results give relevant information about the 0 and 1-regions of $F(I, J)$ from the ones of $I$ and $J$, as Ex. 3.7 will show.

More can be said about the $a$-region of $F(I, J)$ if $a$ is not only idempotent but absorbent, that is,

$$
F(a, x)=F(x, a)=a,
$$

for all $x \in[0,1]$.

Proposition 3.5 Let a be an idempotent element of an aggregation function $F$. Then:

i) $F(I, J)_{a} \supseteq I_{a} \cup J_{a}$ for any $I, J \in \mathcal{I F}$ if and only if a is absorbent.

ii) $F(I, J)_{a}=I_{a} \cup J_{a}$ for any $I, J \in \mathcal{I F}$ if and only if it is $F(x, y)=a$ only when $x=a$ or $y=a$.

\section{Proof.}

i) First, let us suppose $F(I, J)_{a} \supseteq I_{a} \cup J_{a}$.

For any $z \in[0,1]$ we can choose $(x, y), I$ and $J$ such that $(x, y) \in I_{a}$ and $(x, y) \in J_{z}$. Therefore, we have $F(I, J)(x, y)=F(a, z)$. But we know that $F(I, J)(x, y)=a$, because $(x, y) \in$ $F(I, J)_{a}$. That is, $F(a, z)=a$ for any $z$.

In a similar way, we arrive at $F(z, a)=a$, and so $a$ is absorbent.

For the converse, consider $(x, y) \in I_{a} \cup$ $J_{a}$. Then, $F(I, J)(x, y)$ equals $F(a, J(x, y))$ or $F(I(x, y), a)$. In both cases $F(I, J)(x, y)=a$, because $a$ is supposed absorbent, and $(x, y) \in$ $F(I, J)_{a}$

ii) Let us suppose $F(I, J)_{a}=I_{a} \cup J_{a}$.

For $(x, y) \in I_{a} \cup J_{a}$, we have $I(x, y)=a$ or $J(x, y)=a$. Then, $F(x, a)=F(a, x)=a$ because $(x, y) \in F(I, J)_{a}$.

For the converse we need to prove $F(I, J)_{a} \subseteq$ $I_{a} \cap J_{a}$, under the condition $F(x, y)=a$ only when $x=a$ or $y=a$.

Suppose that $(x, y) \in F(I, J)_{a}$. Then, $F(I(x, y), J(x, y))=a$, but this only holds when $I(x, y)=a$ or $J(x, y)=a$, that is, $(x, y) \in I_{a} \cap J_{a}$.

The existence of a neutral element:

$$
F(e, x)=F(x, e)=e,
$$

for all $x \in[0,1]$, has also influence in the aggregation. 
Proposition 3.6 Let $F$ be an aggregation function with neutral element $e \in[0,1]$. Then:

- $F(I, J)(x, y)=J(x, y)$ whenever $(x, y) \in I_{e}$,

- $F(I, J)(x, y)=I(x, y)$ whenever $(x, y) \in J_{e}$.

Proof. For $(x, y) \in I_{e}$, we have:

$$
\begin{aligned}
F(I, J)(x, y) & =F(I(x, y), J(x, y)) \\
& =F(e, J(x, y)) \\
& =J(x, y) .
\end{aligned}
$$

The same for the other case.

Let us conclude with an example.

Example 3.7 We consider two implication functions $I$ and $J$, whose 0 and 1-regions are the shaded areas shown in Fig. 2.

i) If we take as the aggregation function a $t$-norm $T$ with trivial 0-region:

$$
T_{0}=\left\{(x, y) \in[0,1]^{2}: x=0 \text { or } y=0\right\},
$$

then:

- $T$ fulfills the condition in Prop. 3.4-ii for 1 , and so $T(I, J)_{1}=I_{1} \cap J_{1}$.

- As $T(x, y)=0$ only when $x=0$ or $y=0$, we have $T(I, J)_{0}=I_{0} \cup J_{0}$ (Prop. 3.5-ii).

- And according to Prop. 3.6, $T(I, J)=J$ on $I_{1}$ and $T(I, J)=I$ on $J_{1}$ because 1 is neutral.

See Fig. 3.

ii) Similarly if we consider a t-conorm $S$ with trivial 1-region:

$$
S_{1}=\left\{(x, y) \in[0,1]^{2}: x=1 \text { or } y=1\right\} .
$$

In this case, we have:

$-S(x, y)=0$ only for $x=y=0$, and so $S(I, J)_{0}=I_{0} \cap J_{0}$ (Prop. 3.4-ii).

- $S(I, J)_{1}=I_{1} \cup J_{1}$ because $S(x, y)=1$ only when $x=1$ or $y=1$ (Prop. 3.5-ii).

- Finally, Prop. 3.6 applied to the neutral 0 says that $S(I, J)=J$ on $I_{0}$ and $S(I, J)=$ $I$ on $J_{0}$.

Fig. 4 gives the result of the aggregation.

\section{Preservation of properties of implication functions}

Some of the properties that can be required for implication functions are the following. Let $x, y \in$ $[0,1]$ :

(NP) Neutrality principle:

$$
I(1, x)=x,
$$

(CP) Law of contraposition, with respect to a fuzzy negation $N$ :

$$
I(x, y)=I(N(y), N(x)),
$$
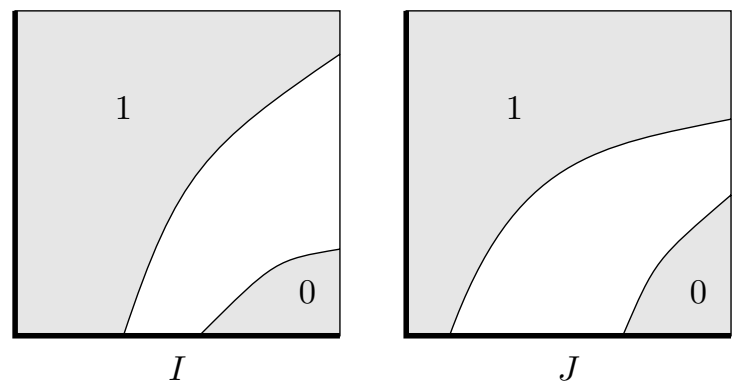

Figure 2: The zero and one regions of $I, J$.

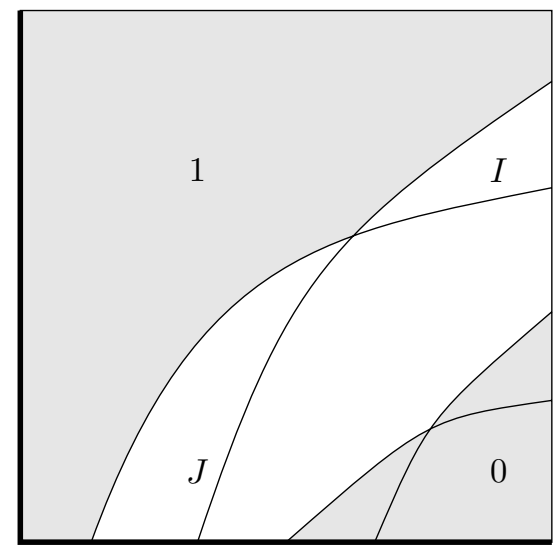

$T(I, J)$

Figure 3: The result of the aggregation for a t-norm $T$ with trivial 0 -region.

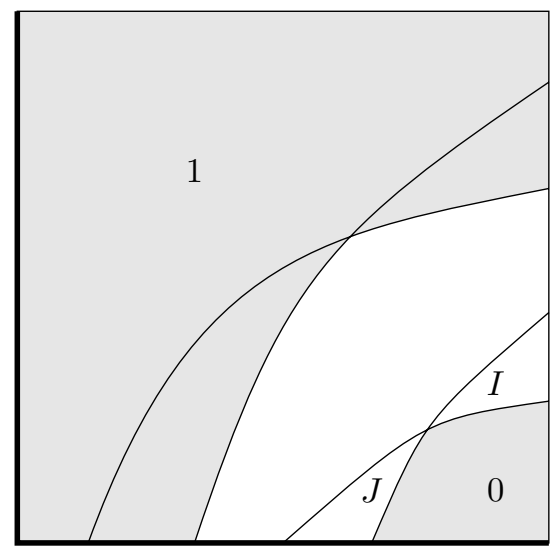

$S(I, J)$

Figure 4: The result of the aggregation for a tconorm $S$ with trivial 1-region. 
(OP) Ordering property:

$$
I(x, y)=1 \Leftrightarrow x \leqslant y
$$

(IP) Identity principle:

$$
I(x, x)=1
$$

(C) Continuity.

Our purpose is to study how $F$ should be in order to preserve those properties. Briefly, we say that $F$ preserves a property $P$ if whatever $I$ and $J$ fulfilling $P$, then $K=F(I, J)$ also satisfies it.

Proposition 4.1 Any aggregation function $F$ preserves $I P$ and $C P$.

Proof. If $I, J$ are IP we have, for all $x \in[0,1]$,

$$
K(x, x)=F(I(x, x), J(x, x))=F(1,1)=1,
$$

and then $K$ also is IP.

For the preservation of $\mathrm{CP}$ :

$$
\begin{aligned}
K( & N(y), N(x)) \\
& =F(I(N(y), N(x)), J(N(y), N(x))) \\
& =F(I(x, y), J(x, y)) \\
& =K(x, y)
\end{aligned}
$$

Proposition 4.2 Let $F$ be an aggregation function. Then:

i) $F$ preserves $N P$ if and only if $F(x, x)=x$ for all $x \in[0,1]$ ( $F$ is idempotent $)$.

ii) $F$ preserves $O P$ if and only if $F(x, y)<1$ when $x, y<1$.

iii) $F$ preserves continuity if and only if $F$ is continuous.

\section{Proof.}

i) If $I, J, K$ are NP we have, for all $y \in[0,1]$,

$$
y=K(1, y)=F(I(1, y), J(1, y))=F(y, y)
$$

then $F$ is idempotent.

For the converse: if $F$ is idempotent and the implications $I, J$ are NP we have, for all $y \in$ $[0,1]$

$$
y=F(y, y)=F(I(1, y), J(1, y))=K(1, y) .
$$

Then, $K$ also fulfills the neutrality principle.

ii) From left-to-right: let us suppose that $F$ preserves OP. Let $a, b<1$. There exist $x, y \in$ $[0,1]$, with $x>y$, and $I, J$ OP such that $I(x, y)=a$ and $J(x, y)=b$. Then,

$$
F(a, b)=F(I(x, y), J(x, y))=K(x, y),
$$

which is less than 1 because $K$ is OP. Now, the converse. The condition is

$$
x, y<1 \Rightarrow F(x, y)<1 .
$$

Let $I, J$ be OP.

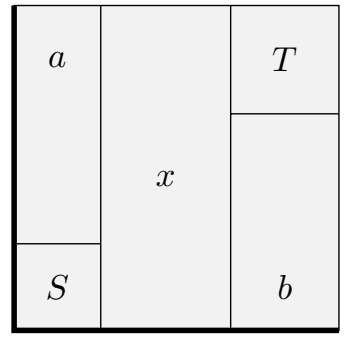

$a<b$

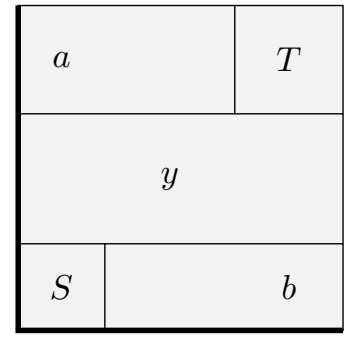

$a>b$
Figure 5: Associative, continuous aggregation functions on $[0,1]^{2}$.

- If $x \leqslant y, I(x, y)=J(x, y)=1$ and then $K(x, y)=F(1,1)=1$

- If $x>y, I(x, y)<1$ and $J(x, y)<1$. So, $K(x, y)<1$ due to the condition.

That is, $K$ is OP and $F$ preserves OP.

iii) The continuity follows directly from the continuity of involved implications $I$ and $J$.

A class of aggregation functions that could be appropriate to our purpose, in order to assure the fulfillness of some of the properties we have dealt with, is that introduced in [3], which can be reformulated as follows.

Proposition 4.3 If $F:[0,1]^{2} \rightarrow[0,1]$ is an associative, continuous in each argument, aggregation function, then $F$ has the form:

$$
F(x, y)= \begin{cases}S(x, y) & \text { if } x, y \in[0, \alpha]^{2} \\ T(x, y) & \text { if } x, y \in[\beta, 1]^{2} \\ (x \wedge y) \vee(x \wedge a) \vee(y \wedge b)) & \text { otherwise. }\end{cases}
$$

where $T, S$ are continuous t-norm and t-norm, $a=$ $F(1,0), b=F(0,1), \alpha=a \wedge b$ and $\beta=a \vee b$.

See Fig. 5, with the two cases $a<b$ and $a>b$.

Taking into account Propositions 4.1 and 4.2, we observe that any continuous associative aggregation function preserves IP, CP, OP and C. With respect to the other property we can establish the following.

Proposition 4.4 A continuous associative aggregation function $F$ preserves NP if and only if it is of the form:

$$
F(x, y)= \begin{cases}x \vee y & \text { if } x, y \in[0, \alpha]^{2} \\ x \wedge y & \text { if } x, y \in[\beta, 1]^{2} \\ (x \wedge y) \vee(x \wedge a) \vee(y \wedge b)) & \text { otherwise. }\end{cases}
$$

Proof. The condition for NP is the idempotency of $F$. So, $T=\min$ and $S=\max$ in (3). 

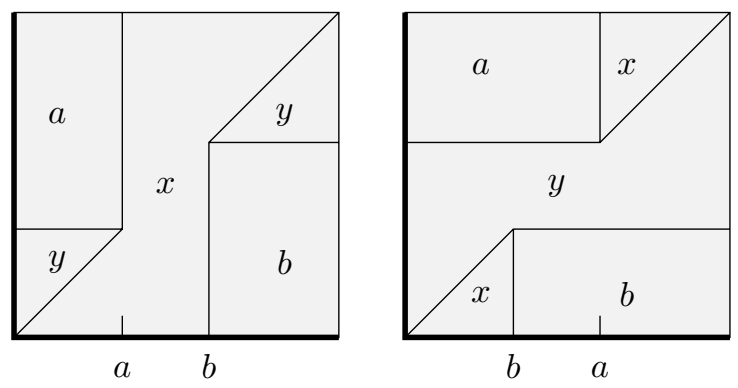

Figure 6: Associative, continuous, idempotent aggregation functions on $[0,1]^{2}$.

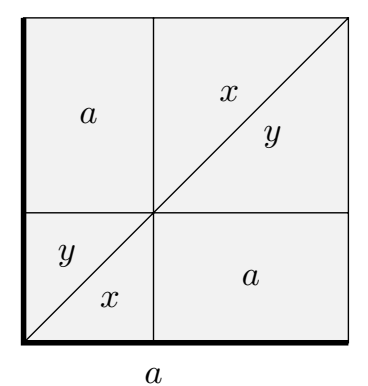

Figure 7: Associative, continuous, idempotent, commutative aggregation functions on $[0,1]^{2}$.

The two cases $a<b$ and $a>b$ can be seen in Fig. 6.

Remark 4.5 A continuous associative aggregation function $F$ is commutative if and only if $a=b$. In this case the aggregation functions, shown in Fig. 7, are the following:

$$
F(x, y)= \begin{cases}x \vee y & \text { if } x, y \in[0, \alpha]^{2} \\ x \wedge y & \text { if } x, y \in[\beta, 1]^{2} \\ a & \text { otherwise }\end{cases}
$$

\section{Conclusions}

Firstly, we have described how a binary operation on the unit interval $[0,1]$ should be in order to transform two implication functions in another implication function.

We have considered some aspects of the aggregated implication functions in terms of the two given ones and also we have analysed under which conditions the aggregation operation preserves some significant properties of the involved implication functions.

\section{Acknowledgements}

The authors acknowledge the support of the Spanish DGI grants MTM2009-10962, TIN2009-07901 and TIN2012-32482.

\section{References}

[1] M. Baczynski, B. Jayaram, Fuzzy Implications. London: Springer, Studies in Fuzziness and Soft Computing vol. 231, 2008.

[2] E. P. Klement, R. Mesiar and E. Pap, Triangular Norms. Dordrecht: Kluwer Academic Publisher, 2000.

[3] J.L. MARIChal, Aggregation operators for multicriteria decision aid. Ph.D. thesis, Institute of Mathematics, University of Liege, Belgium, 1999.

[4] S. Massanet, G. Mayor, R. Mesiar, J. TORRENS On fuzzy implications: an axiomatic approach. International Journal of Approximate Reasoning (to appear) 